\title{
The top 10 things to know about transfusion medicine before intern year: an evidence-based course for graduating medical students
}

\author{
Alexis R. Peedin*, Irina Perjar, Marshall A. Mazepa ${ }^{\dagger}$ Marian A. Rollins-Raval", Yara A. Park, \\ Jay S. Raval \\ Department of Pathology and Laboratory Medicine, University of North Carolina, Chapel Hill, NC, USA
}

p-ISSN 2287-979X / e-ISSN 2288-0011
https://doi.org/10.5045/br.2019.54.2.125
Blood Res 2019;54:125-130.

Received on January 29, 2019

Accepted on March 8, 2019

Current Affiliation

*Department of Pathology, Anatomy, and Cell Biology, Thomas Jefferson University Hospital, Philadelphia, PA, USA

Department of Medicine, University of Minnesota, Minneapolis, MN, USA

${ }^{\#}$ Department of Pathology, University of New Mexico, Albuquerque, NM, USA

\section{Correspondence to}

Alexis R. Peedin, M.D.

Department of Pathology, Anatomy, and Cell Biology, Thomas Jefferson University Hospital, 111 South 11th St., Room 8220, Gibbon Building, Philadelphia, PA 19107, USA

E-mail: Alexis.Peedin@jefferson.edu

(C) 2019 Korean Society of Hematology

\section{Background}

Transfusion medicine (TM) knowledge varies widely among physician trainees. In addition, there have been few instances in which curricular changes have been meaningfully assessed for TM education in medical school.

\section{Methods}

We created and presented a novel lecture to improve TM knowledge for graduating medical students using eight objectives designed to reinforce critical information about blood management. Each objective was coded according to unique color schemes, fonts, and graphics to create visual associations while quickly and clearly presenting complex concepts. The validated BEST Collaborative exam was used to measure changes in student TM knowledge, while a survey was conducted to gauge changes in confidence for each objective. Students were asked to submit anonymous feedback about their experiences.

Results

The mean student post-course exam score was $50.0 \%$, while the pre-course baseline score was $27.5 \%(P<0.0001)$. Mean confidence levels increased significantly for all objectives. Student feedback was universally positive.

\section{Conclusion}

This study improved knowledge and confidence for graduating medical students by utilizing engaging and visually stimulating presentations to display high-impact TM material. However, further efforts are needed to optimize learning.

Key Words Education, Medical student, Transfusion, Residency

\section{INTRODUCTION}

Transfusion medicine (TM) knowledge varies widely among resident physicians in both the theoretical and practical contexts $[1,2]$. This can lead to the inappropriate utilization of blood products, thereby increasing both the costs and risk of adverse events posed to patients. Our academic medical center is also subject to these problems; many orders received at our blood bank do not align with evidence-based national transfusion guidelines. American medical schools lack standardized TM educational programs. There is also great variability in the number of hours and settings in which TM is taught [3]. Thus, residents and fellows are often left to learn TM principles in actual applied settings while caring for patients with blood product needs.
The most recent dedicated TM curriculum for medical students was published in 1995 by the Transfusion Medicine Academic Awards (TMAA) group and sponsored by the National Heart, Lung, and Blood Institute. This effort represented an update to the curricula previously published in 1983 and 1989 (hereinafter referred to as the TMAA curriculum) [4]. While the group has since disbanded, the Academy of Clinical Laboratory Physicians and Scientists (ACLPS) proposed another curriculum that was published in 2010 in the context of general laboratory medicine education for medical students [5]. There were many challenges in implementing each of these proposed curricula, with few publications detailing the development of structured programs. One recent successful program involved case-based patient simulations [6]. Other institutional attempts to increase general laboratory medicine education for medical students have 
received mixed feedback from students. One study found that only $41 \%$ of graduating students who participated in a 1.5-day course (based on the 2010 ACLPS curriculum) agreed that laboratory medicine would be useful for their future practices [7]. This contrasts with findings suggesting that more than $70 \%$ of all clinical decisions are guided by laboratory tests [8]. It is thus of high importance to induce a major shift in the level of appreciation medical students have for the indispensable nature of laboratory medicine education, including TM.

It can be very challenging to meaningfully assess the curricular changes related to this type of education. Here, experience in the field of TM has been particularly scant. In 2014, the Biomedical Excellence for Safer Transfusion (BEST) Collaborative developed and validated an exam designed to assess the knowledge of medical students and physicians (hereinafter referred to as the BEST exam) [9]. The BEST exam was validated for use among test-takers equipped with expected basic, intermediate, and advanced TM knowledge, ranging from first-year medical residents to TM physicians. Some publications have recently begun utilizing the BEST exam to assess post-graduate medical trainee knowledge [10, 11].

In an effort to support TM education for senior medical students, we developed a novel course based on the 1995 TMAA curriculum within our medical school. The course covers the most practical aspects of the TMAA curriculum, targets indications for blood product transfusions, presents scenarios requiring special blood product modifications, and requires the identification and management of transfusion reactions. This article thus reports on our creation of this one-hour course, which should easily be adaptable at other institutions.

\section{MATERIALS AND METHODS}

\section{Study population}

Medical students at our public institution in the Southeastern United States received a one-hour lecture and two hours of small groupwork dedicated to the introductory principles of TM during their pre-clinical years. In 2015, all 180 graduating medical students were invited to attend this additional and optional one-hour pilot course, which was offered as part of a one-week lecture series that took place approximately one month after resident Match Day.

\section{Planning the course}

The scope of the course was created by selecting eight of 28 objectives from the TMAA curriculum that the school's laboratory medicine educational faculty felt were crucial for new residents. These eight objectives were then partitioned to create a lecture titled "Top 10 Things to Know About Transfusion Medicine Before Intern Year" (Table 1). The contents were designed to be both patient and resident-centered, thus positing each objective as a question from the perspective of a physician caring for a patient in real-time. The class was presented in a lecture hall by a TM faculty member. Another faculty member and a senior pathology resident answered questions both during and after the course.

We designed slides to distinctly differ from the typical bullet-point format by using a website that allowed users to create infographics (www.piktochart.com). Each objective featured a unique color scheme and font. These elements were also accompanied by a variety of graphics that quickly and clearly presented complex concepts while sustaining student engagement and creating visual associations (Fig. 1). Minimal data were presented on each slide as the instructor verbally described relevant background information. Slide contents were based on national evidence-based guidelines in addition to being adapted from lectures previously given by TM faculty at our institution.

\section{Educational activities and student evaluations}

After selecting the course contents, we obtained and reviewed the BEST exam to ensure content overlap. Our medical students took the BEST exam immediately after completing the course and were not allowed to keep their test materials. We first considered administering the exam prior to the course to obtain a pre-intervention baseline specific to our study population. However, possible disadvantages were associated with students seeing the exam before our novel educational intervention. Students were thus not offered the exam prior to the start of the presentation (see the Comparison Group section below). Student confidence levels in performing tasks related to the course objectives

Table 1. "The top 10 things to know about transfusion medicine before intern year" lecture objectives for the UNC transfusion medicine capstone course 2015.

1. When does my patient need red blood cells?

2. When does my patient need other blood products? - Platelets, plasma, cryoprecipitate, granulocytes

3. When does my patient need specially modified blood products?

- Leukoreduced, irradiated, washed, Hemoglobin S negative

4. What tests should I order before giving my patient blood products?

- Type, screen, crossmatch

5. My patient can't wait for crossmatched blood! What can I do? - Emergency and conditional release blood

6. What is the risk of blood-borne disease transmission? - Bacteria, parvovirus B19, Hepatitis B, Hepatitis A, Hepatitis C, HIV, HTLV-I and -II

7. Transfusion reactions: What is common? What is severe? - Allergic, febrile non-hemolytic, acute hemolytic, transfusionrelated acute lung injury (TRALI), transfusion-associated circulatory overload (TACO)

8. What is massive transfusion protocol, and what do I do when my patient is on it?

9. Which patients might benefit from apheresis?

10. What do I do when I don't know what to do? - Call the blood bank to talk to a resident, fellow, or attendant on call at any hour of any day 


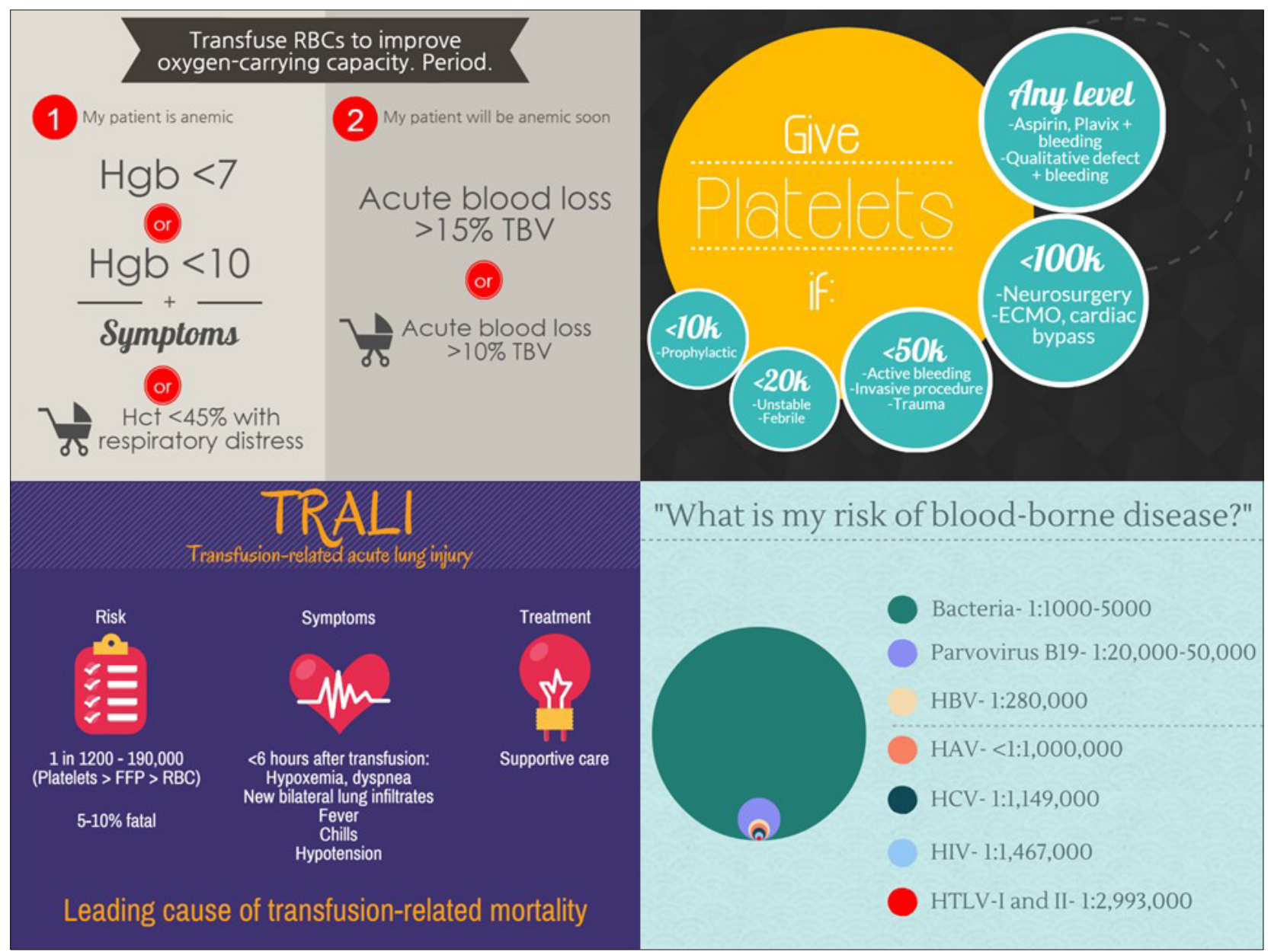

Fig. 1. Representative slides from the UNC blood banking/transfusion medicine review course 2015.

were assessed with institutional review board approval immediately before and after the course based on self-evaluations of their perceived efficacy. These self-efficacy surveys implemented a confidence score ranging from 0-10 (with 0 indicating "cannot do at all," 5 indicating "moderately certain can do," and 10 indicating "highly certain can do") [12]. The medical school then solicited anonymous student feedback about the course by providing email invitations to a corresponding online survey.

\section{Comparison group}

We intended to characterize the impact of our novel course on medical student TM knowledge as accurately as possible. We therefore needed to obtain a relevant and meaningful pre-intervention baseline specific to our study population to fully characterize the impact of our institution's standard curriculum on TM knowledge before students attended our lesson. We thus offered our course the following year (2016) approximately one month after Match Day. All attending fourth-year medical students were given the BEST exam prior to the start of our presentation. As the standard four-year curriculum had not changed at our medical in- stitution, we deemed it reasonable to use this group of students for comparison. All related study elements were approved by our Institutional Review Board prior to conducting the course (\#15-0906).

\section{Statistical analysis}

Our study population's post-course BEST exam scores were compared to the comparison group's pre-course BEST exam scores using the two-tailed Mann-Whitney test. Changes in confidence scores for each objective were compared using the two-tailed Wilcoxon matched paired test for each student in the study population who completed both the pre-course and post-course self-efficacy surveys. Data were analyzed using GraphPad Prism 6.0 (GraphPad Software, Inc., La Jolla, CA). Statistical significance was defined as $P \leq 0.05$.

\section{RESULTS}

From our study population, 63 students (35\% of the total graduating class) attended the course. Of them, 62 (98.4\%) completed the BEST exam and self-efficacy assessments after 
the lesson was completed. The mean exam score was $50 \%$, while the median score was $50 \%$. The 25 th and 75 th percentiles were $43.8 \%$ and $60 \%$, respectively. For the comparison group evaluated the following year, 91 students $(50.6 \%$ of the total graduating class) attended our course. Of them, $88(96.7 \%)$ completed the BEST exam prior to the start of the class. This comparison group was used as our pre-intervention baseline. Here, the mean exam score was $27.5 \%$, while the median score was $25 \%$, and the 25 th and 75 th percentiles were $15 \%$ and $35 \%$, respectively. Results indicated a significant post-intervention increase in overall TM knowledge $(P<0.0001)$.

Confidence levels rose significantly for all objectives among our study population (i.e., from a mean of 3.1 to 7.0) $(P<0.0001)$ (Table 2). Student answers to the BEST exam were evaluated to assess performance in the tested topics. Students performed poorly for three of the 20 questions, with fewer than $20 \%$ of respondents answering correctly. These topics included the diagnosis and management of allergic transfusion reactions (10\% answered correctly), red cell transfusion thresholds in acute anemia $(11 \%$ answered correctly), and massive transfusions (11\% answered correctly). The remaining questions were answered correctly by an average of $56.2 \%$ of students in the study population (ranging from $26-82 \%$ ).

Out of the total 63 students in our study population, 53 $(84.1 \%)$ course attendants submitted anonymous evaluations to the medical school. Here, ratings were given for content presentation clarity, session organization, active learning opportunities, and the overall session. Items were rated on a scale of 1-5 (1=not at all/poor, 3=somewhat/good, 5=com- pletely/excellent); all parameters were rated at 4.7 or above. Students universally found the curriculum enjoyable, relevant, effective, and time-efficient. Some representative comments are as follows: "This session was very clear and well-run. The slides were fantastic!", and "A gem of a lecture - I think this should be a required lecture for all 4th year medical students - absolutely the most bang for your buck in a single hour".

\section{DISCUSSION}

This study was an initial effort to assess the impact of a novel TM course by conducting a validated exam on medical student TM knowledge. Our findings demonstrated that, immediately after completing the course, graduating medical students possessed significantly improved TM knowledge between the previously reported basic and intermediate levels associated with the BEST exam [9]. Fewer than 20\% of all students correctly answered three questions on the post-course BEST exam (i.e., diagnosis and management of allergic transfusion reactions, red cell transfusion thresholds in acute anemia, and massive transfusions). We thus modified our educational plan to include additional lecture time on these areas and expanded the question-and-answer session to improve student understanding.

The medical students that attended our lecture significantly increased their confidence levels in performing TM-related tasks. Notably, the three topics most poorly answered received some of the highest self-reported confidence ratings based on post-course evaluations, ranging from 7.2

Table 2. Self-Efficacy Survey results for the UNC transfusion medicine capstone course 2015.

\begin{tabular}{|c|c|c|c|}
\hline & $\begin{array}{c}\text { Pre-capstone } \\
\text { confidence }(0-10)\end{array}$ & $\begin{array}{c}\text { Post-capstone } \\
\text { confidence }(0-10)\end{array}$ & $P$ \\
\hline \multicolumn{4}{|l|}{ Appropriately prescribing blood products } \\
\hline A. Packed red blood cells & 5.9 & 7.6 & $<0.0001$ \\
\hline B. Platelets & 4.8 & 7.2 & $<0.0001$ \\
\hline C. Plasma products (FFP/FP24) & 4.1 & 7.0 & $<0.0001$ \\
\hline D. Cryoprecipitate & 3.5 & 6.8 & $<0.0001$ \\
\hline E. Granulocytes & 1.9 & 5.8 & $<0.0001$ \\
\hline \multicolumn{4}{|l|}{ Appropriately ordering specially modified blood products } \\
\hline A. Leukoreduction & 1.9 & 7.5 & $<0.0001$ \\
\hline B. Washing & 2.1 & 7.1 & $<0.0001$ \\
\hline C. Irradiation & 2.0 & 7.1 & $<0.0001$ \\
\hline \multicolumn{4}{|l|}{ Appropriately prescribing blood products in special situations } \\
\hline A. Massive transfusion protocol & 3.2 & 7.2 & $<0.0001$ \\
\hline B. Warm-reacting autoantibody & 1.6 & 5.8 & $<0.0001$ \\
\hline C. Type \& screen results not available & 3.2 & 7.3 & $<0.0001$ \\
\hline \multicolumn{4}{|l|}{ Appropriately identifying and managing transfusion reactions } \\
\hline A. Allergic & 4.2 & 7.5 & $<0.0001$ \\
\hline B. Febrile non-hemolytic & 3.6 & 7.1 & $<0.0001$ \\
\hline C. Acute hemolytic & 3.0 & 6.8 & $<0.0001$ \\
\hline D. Transfusion-related acute lung injury (TRALI) & 2.4 & 6.6 & $<0.0001$ \\
\hline E. Transfusion-associated circulatory overload (TACO) & 2.2 & 7.0 & $<0.0001$ \\
\hline
\end{tabular}


to 7.6 out of 10 . This suggests that this metric is a non-specific measure of improved understanding. Self-assessments are widely acknowledged to be ineffective and inaccurate methods for identifying personal areas of deficiency [13, 14]. Thus, while self-assessment confidence ratings significantly improved for all objectives after our course, these self-reported results should be interpreted wisely. External assessments also remain vital when attempting to accurately characterize student understanding of education materials.

Since our course contents were based on national evidence-based guidelines and were not institution-specific, the lecture can easily be adapted for use at other medical schools. This course would best be applied at medical schools that dedicate minimal instruction time for TM education. This is because it covers highly practical aspects of transfusion in only one hour. It may be challenging to adapt this course at medical schools that do not have TM physicians on staff to provide routine instruction. The slides used in the complete presentation are available from the corresponding author by request.

This study was somewhat limited in that we were not able to perform a pre-intervention assessment of our study population prior to the lesson. As already noted, we did not want to administer the validated BEST exam prior to the course to avoid biasing the assessment of respondent TM knowledge immediately afterward. However, we believe that assessing the fourth-year medical student group at our course the following year in addition to characterizing their TM knowledge via the BEST exam prior to the lesson to serve as our pre-intervention baseline was a reasonable solution to this dilemma. It is reasonable to assume that the fourth-year medical students in both the study population and in the comparison group had identical levels of TM knowledge. This is because both groups completed identical medical education curricula for four-year periods prior to taking our course and were accordingly tested at corresponding progress levels (i.e., approximately one month after Match Day). We thus concluded that this comparison group could equitably serve as a pre-course baseline for our students.

We plan to repeat our course for subsequent groups of graduating medical students. The positive student reactions were highly encouraging, particularly since many attempts to teach laboratory medicine to medical students are met with poor feedback. However, increased attention will be given to the previously mentioned topics for which students performed most poorly on the exam. In addition, we plan to immediately create and include an informational handout, which some students requested. We did not initially provide a student handout because participants were offered the post-course BEST exam. That is, there were concerns that it may have provided a source of information that would have artifactually increased post-course scores. However, other institutions that intend to adopt this course may consider providing a handout to students for reference in their future practices.

Future studies assessing longer-term retention of the mate- rial covered in this course have already been planned. We also plan to adapt this course for use in a required online training module for all new residents. This will reach a broader audience, thereby improving the blood ordering practices at our institution. In addition, we intend to implement this novel course among several resident groups at various training levels, including those related to internal medicine, surgery, pediatrics, and both adult and pediatric hematology/oncology and nephrology fellows. We anticipate that periodic exposure to this course will be necessary to continually reinforce core TM objectives for physicians who will care for patients with blood product needs.

\section{ACKNOWLEDGMENTS}

The authors wish to thank the following persons and entity for their help with this research: 1) Mrs. Johanna Foster, Director of Curriculum Support at the University of North Carolina School of Medicine (Chapel Hill, NC), for her assistance in and support of this initiative, 2) Dr. Peadar Noone, Professor of Medicine and Course Director of the University of North Carolina School of Medicine Capstone Course Series, for his support of this initiative, 3) Dr. Richard Haspel, Associate Professor of Pathology at the Beth Israel Deaconess Medical Center (Boston, MA), and 4) the BEST Collaborative for providing their validated transfusion medicine examination and answer key.

\section{Authors' Disclosures of Potential Conflicts of Interest}

No potential conflicts of interest relevant to this article were reported.

\section{REFERENCES}

1. Bryant BJ, Alperin JB, Indrikovs AJ. Blood bank on-call physician's experiences at a large university medical center. Transfusion 2005;45:35-40.

2. O'Brien KL, Champeaux AL, Sundell ZE, Short MW, Roth BJ. Transfusion medicine knowledge in Postgraduate Year 1 residents. Transfusion 2010;50:1649-53.

3. Karp JK, Weston CM, King KE. Transfusion medicine in American undergraduate medical education. Transfusion 2011;51:2470-9.

4. Cable RG, Thal SE, Fink A, Calhoun L, Petz LD. A comprehensive transfusion medicine curriculum for medical students. Transfusion Medicine Academic Award Group. Transfusion 1995;35:465-9.

5. Smith BR, Aguero-Rosenfeld M, Anastasi J, et al. Educating medical students in laboratory medicine: a proposed curriculum. Am J Clin Pathol 2010;133:533-42.

6. Morgan S, Rioux-Masse B, Oancea C, Cohn C, Harmon J Jr, Konia M. Simulation-based education for transfusion medicine. Transfusion 2015;55:919-25.

7. Molinaro RJ, Winkler AM, Kraft CS, et al. Teaching laboratory 
medicine to medical students: implementation and evaluation. Arch Pathol Lab Med 2012;136:1423-9.

8. American Clinical Laboratory Association. Clinical laboratory testing: Life saving medicine starts here. Washington, DC: American Clinical Laboratory Association, 2007. (Accessed December 20, 2018, at https://www.acla.com/wp-content/ uploads/2013/12/ACLA_Overview_OneSheet_v07.pdf).

9. Haspel RL, Lin Y, Fisher P, Ali A, Parks E; Biomedical Excellence for Safer Transfusion (BEST) Collaborative. Development of a validated exam to assess physician transfusion medicine knowledge. Transfusion 2014;54:1225-30.

10. Haspel RL, Lin Y, Mallick R, et al. Internal medicine resident knowledge of transfusion medicine: results from the BEST-TEST international education needs assessment. Transfusion 2015;
$55: 1355-61$

11. Lin Y, Tinmouth A, Mallick R, Haspel RL; BEST-TEST2 Investigators. BEST-TEST2: assessment of hematology trainee knowledge of transfusion medicine. Transfusion 2016;56:304-10.

12. Urdan T, Pajares F. Self-efficacy beliefs of adolescents. Charlotte, NC: Information Age Publishing, 2006:307-37.

13. Davis DA, Mazmanian PE, Fordis M, Van Harrison R, Thorpe KE, Perrier L. Accuracy of physician self-assessment compared with observed measures of competence: a systematic review. JAMA 2006;296:1094-102.

14. Regehr G, Eva K. Self-assessment, self-direction, and the self-regulating professional. Clin Orthop Relat Res 2006;449: 34-8. 